\title{
A Short Term Comparative Study Between Lichtenstein Technique and Mesh-with-Plug Technique in Management of Inguinal Hernia
}

\author{
Ashraf H. Abbas ${ }^{*}$, Hatem Hussain \\ Department of Surgery, Faculty of Medicine, Suez Canal University hospital, Egypt
}

\begin{abstract}
Background: Repair of groin hernias has evolved from primary tissue repairs, which have a tendency of recurrence. Open mesh used in inguinal hernia repair can be configured as a flat patch (Lichtenstein operation) or as a cone-shaped plug (Mesh with Plug operation). Aim: To compare between Lichtenstein patch hernioplasty and mesh plug hernioplasty to find out which of them is easier to apply and rate of post operative complications in both of them. Methods: sixty patients were randomly allocated to receive either a Lichtenstein patch or a Mesh with Plug. Data of postoperative day 1, 4 weeks and six months were followed up to include operating time, postoperative pain, analgesic medication, return to activity and work. Follow up period was six months. Results: Operating time (mean $=61.53$ vs. 75.17 minutes) was significantly shorter in Lichtenstein group $(P=0.001)$. During day 1 , Patients who had undergone the Mesh with Plug operation experienced more pain rather than Lichtenstein group $(P=0.001)$. Number of retrieved analgesic medication was significantly greater in Lichtenstein group (mean $=15 \pm 3.43$ vs. 12.4 \pm 3 ). Hospital stay and Sick-leave period were similar in both groups. No recurrence rates after six months follow up. Conclusions: Compared with patients received Mesh Plug, patients who underwent Lichtenstein operation experienced less postoperative pain in 24 hour, but consumed little analgesic medication. The rate of return to work is similar in both groups, which indicates no superiority for both. There is no recurrence rate after six months follow up.
\end{abstract}

Keywords: hernioplasty, groin, ligation, recurrence

\section{Introduction}

Inguinal hernias are common in both men \& women and as a result of weakening of the tissue in the groin due to stress or aging $^{(1)}$. The master was Bassini (1885) who introduced a radical cure for inguinal her$\mathrm{nia}^{(2)}$ allowing high ligation of the hernia sac and split fascia was reconstructed along with the transversus aponeurosis and internal oblique down to the inguinal ligament ${ }^{(3)}$. Other traditional tissue-based techniques (e.g., McVay, Shouldice) characterized the armamentarium of the inguinal hernia surgeon during the 1970 s and early $1980 s^{(4)}$. With the need to reduce the rate of hernia recurrence, as well as postoperative pain and convalescence, the treatment of inguinal hernias underwent a dramatic evolution over the past 25 years. The major advances included the introduction of the concept of tension-free hernia surgery, the 
use of prosthetic materials, and the development of laparoscopic techniques ${ }^{(5)}$. Its described advantage was that it requires minimal dissection and has a quicker learning curve. It is promoted as a repair that can be easily done under local anesthesia. Gilbert introduced the Mesh plug Repair system based on input from Usher, which is a suture less repair of inguinal hernia with an umbrella plug along with an on lay patch was an attempt in similar direction ${ }^{(6)}$. Lichtenstein tension free repair introduced in 1986 opened a new era in groin hernia repair with superior results over prior methods, in this repair the inguinal canal is approached from an open anterior approach $^{(7)}$. Thus the use of mesh in hernia repairs however was not widely accepted for use until Lichtenstein introduced the tension free repair ${ }^{(8)}$. During the same period in the early 1990s, Rutkow and Robbins developed the plug mesh technique. Their repair used an umbrella plug held together by suture ${ }^{(9)}$. Statistical studies of medical problems are very important as they can determine the size of the problem and help the health authorities to put appropriate plans in place to deal with them, especially for congenital conditions, as incidence of inguinal hernia ranges between $0.8 \%$ and $4.4 \%^{(10)}$. This study was conducted aiming at comparing Lichtenstein patch hernioplasty and mesh plug hernioplasty regarding intraoperative and early postoperative advantages, complications and recurrence rate.

\section{Patients and Methods}

This study was performed in El-Kantra East hospital and Ismailia University Teaching Hospital. Sixty patients were divided into two equal groups, by consecutive randomization, for the 2 open techniques of repair. Patients with criteria of an uncomplicated unilateral inguinal hernia were selected for the study. Exclusion criteria were patients with predisposing factors for recurrence, complicated inguinal hernia, congenital or recurrent hernia. Patients then received physical examination and preoperative investigations (urine analysis, stool analysis, complete blood picture with differential leucocytic count, random blood sugar, blood urea, serum creatinine, ECG and chest X-Ray) consistent with their physical status and were provided with standardized sheets of written information normally given to patients who are being treated in the hospital. All patients gave written informed consent. The surgical techniques that have been used were those described by $\mathrm{Amid}^{(7)}$ for the Lichtenstein method and by Gilbert and Rutkow ${ }^{(11)}$ for the Mesh Plug method. For Lichtenstein repair: Mesh prosthesis, Prolene ${ }^{T M}$ mesh, Ethicon ${ }^{\circledR} 6 \times 11$ $\mathrm{cm}$ was positioned over the inguinal floor. The medial end was secured to the anterior rectus sheath at least $2 \mathrm{~cm}$ medial to the pubic tubercle. Then, a slit was made at the lateral end of the mesh in such a way as to create two tails which were positioned around the cord structures to create a shatter valve. Finally, the prosthesis was secured to the underlying internal oblique muscle and rectus fascia ${ }^{(12)}$. For Mesh with Plug repair: The plug was folded from a half standard sized $15 \times 15 \mathrm{~cm}$ sheet of flat prolene mesh (Prolene ${ }^{T M}$ mesh, Ethicon ${ }^{\circledR}$ ) as recommended by Rutkow and Robbins $^{(13)}$. The polypropylene cone or "plug" was placed into the direct opening such that its rim was directly flush with the transversalis floor. Multiple interrupted oo absorbable sutures could be used to secure the perimeter of cone to the transversalis tissues. Finally the onlay "patch" of polypropylene mesh was placed over the entire direct floor in the same manner as described in the preceding Lichtenstein repair. The two tails of mesh were joined together producing the new internal ring to support the potentially weakened deep 
ring in direct hernia and the potentially weakened direct space in indirect hernia ${ }^{(14)}$. All patients at the discharge were instructed and informed about the post operative complications that will be recorded in the follow up visits. A wound infection was defined as the presence of a purulent discharge from the operation site. All patients were provided with instructions to complete and record Visual Analogue Scores (VASs) ${ }^{(15)}$ for pain assessment during the first 24 hours after the operation. In addition, the number of analgesics after the operation that each patient took was recorded. Patients attended the hospital on day 7 and after 4 weeks. For assessment of post-operative complications, further fol- low-up was performed at 6 months to record the return to normal activity and work and if there is any recurrence.

\section{Results}

Sixty patients were enrolled in this study, their age ranged from 22-67 years. Age and employment were described in table (1). In Mesh Plug group 13 patients suffered from indirect inguinal hernia (43.3\%) and direct type were found in 17 patients (56.7\%) where indirect type were found in 22 patients $(73.3 \%)$ and direct type were found in 6 patients (20\%) with pantaloon type found in 2 patients $(6.7 \%)$ in Lichtenstein group of patients table (1).

Table 1: Patients characteristics

\begin{tabular}{|l|lll|}
\hline & Lichtenstein & Plug and patch & $P$ value \\
\hline Age (year; mean \& range) & $44.8(24-67)$ & $43.6(22-64)$ & 0.713 \\
\hline Employment & & & 0.490 \\
$\quad$ Employed & $21(70)$ & $25(83.3)$ & \\
Self-employed & $2(6.7)$ & $1(3.3)$ & \\
Retired & $7(23.3)$ & $4(13.3)$ & \\
\hline Type of hernia & & & 0.072 \\
Direct & $6(20)$ & $17(56.7)$ & \\
Indirect & $22(73.3)$ & $13(43.3)$ & \\
$\quad$ Pantaloon type & $2(6.7)$ & 0 & 0.001 \\
\hline Operating time (min), mean (range) & $62(45-77)$ & $75(60-90)$ & 0.775 \\
\hline Hospital stay (hrs), mean (range) & $44(24-60)$ & $45(24-72)$ & \\
\hline
\end{tabular}

Data are presented as no (\%)

Hospital stay period was similar in both groups of mesh plug and Lichtenstein techniques with mean value was $45 \pm 14.09$ hours and $44 \pm 10.08$ hours respectively; the difference between both groups was statistically insignificant table (1). Operative time was shorter in Lichtenstein group, its mean value was $61.53 \pm 9.86$ minutes compared to mesh plug group whose mean value was $75.17 \pm 6.66$ minutes, the difference between both group was statistically significant $(P$ value $=0.001)$ table $(1)$. One patient in Mesh with Plug group developed cord swelling and 2 developed hematoma while in Lichtenstein group only 1 patient developed hematoma with no cord swelling which subsided in period of 10 days (Table 2). Two patients in Mesh with Plug group developed post operative fever, while in Lichtenstein group only 1 patient developed post operative fever which is due to chest infection which responded to antibiotics. During day 1 post operatively, pain score was greater in mesh plug group, its mean value was $6.77 \pm 2.08$, compared to Lichtenstein group whose mean value was 
$4.83 \pm 1.09$, the difference between both group was statistically significant $(P$ value= 0.001). Sick-leave period was similar in both groups of mesh plug and Lichtenstein techniques with mean value of $17 \pm 4.44$ days and $16 \pm 3.89$ days respectively; the difference between both groups was statistically insignificant. Numbers of analgesic medication received was lower in mesh plug group, its mean value was $12.4 \pm 3$, compared to Lichtenstein group, whose mean value was $15 \pm 3.43$, the difference between both group was statistically significant $(P$ value $=0.001)$ (Table 2$)$. No recurrence rates in both groups for follow up of 6 months.

Table 2: Post-operative complications and post-operative pain.

\begin{tabular}{|l|lll|}
\hline & Lichtenstein & Plug and patch & $P$ value \\
\hline Cord swelling & 0 & 1 & 0.450 \\
Fever & 1 & 2 & 0.610 \\
Hematoma & 1 & 2 & 0.610 \\
Seroma & 1 & 1 & 1.00 \\
Superficial wound infection & 1 & 0 & 1.00 \\
Hydrocele & 0 & 1 & 0.490 \\
\hline Visual analogue scale (mean, range) & $4.8(2-9)$ & $6.7(2-10)$ & 0.001 \\
\hline Sick-leave period (days; mean and range) & $16(8-21)$ & $17(11-30)$ & 0.221 \\
\hline Numbers of retrieved analgesics & $15(0-20)$ & $12(8-20)$ & 0.005 \\
(Pills; mean range) & & & \\
\hline Short term recurrence & 0 & 0 & 0.001 \\
\hline Data are presented as no (\%) & & &
\end{tabular}

\section{Discussion}

The Lichtenstein inguinal hernia repair was the first pure prosthetic, tension-free repair to achieve consistently low recurrence rates in long-term outcomes analysis ${ }^{(12)}$. Rutkow and Robbins have reported interesting and effective advances in the Lichtenstein technique. The "Mesh with Plug" repair, represents a tension-free herniorrhaphy and can even be performed with or without sutures $^{(16)}$. In this study, we aimed to compare between these two techniques as regard: The primary outcome was operative technique, operative time, hospital stay, convalescence (or sick-leave) period and early and late postoperative complications. Secondary outcomes included the recurrence rate 6 months after surgery. We found that there is a high significant difference between both groups in duration of surgery. Mesh with Plug technique was longer (mean value was 75.17 minutes) due to time needed to apply the plug in the defect and its fixation with sutures. In Lichtenstein technique, the mean was 61.53 minutes, due to only fixation of the patch to the entire floor of the inguinal canal after repairing the defect. While the operative time, statistically insignificant with Saad et $\mathrm{al}^{(17)}$, was 38 minutes (median) for Mesh Plug compared to 43 minutes (median) for Lichtenstein; and the significance in operative time was proved also by Horharin et $\mathrm{al}^{(18)}$ an Kingorth et $\mathrm{al}^{(19)}$, (median $=60$ minutes for Mesh with Plug compared to median= 82 for lichtenstein) and (median= 31 minutes for Mesh with Plug compared to median= 37 for lichtenstein) respectively. The short time reported by previous studies is explained by that they did not apply fixation of the patch with sutures in their all operations after plug placement, which shorten the operative time needed for 
Mesh Plug technique. Pain analogue score was significantly higher in post mesh plug repair (median $=6.7$ ) versus Lichtenstein repair (median $=4.8$ ) at 24 hours postoperatively. This finding is not matched with Nienhuijs et $\mathrm{al}^{(20)}$ and Saad et $\mathrm{al}^{(17)}$, who reported rapid discharge of patients with minimum postoperative pain in cases dealt with mesh plug technique rather than Lichtenstein technique. This could be explained in our study by the more extensive dissection of the posterior inguinal floor added to the plug fixation by interrupted sutures. This could be the reason of decreased number of analgesic tablets used in the outpatient with mean of 15 of retrieved tablets in group of Lichtenstein group versus the increased number of used analgesic tablet used in the outpatient with the mean of 12.4 of retrieved tablets in group of mesh plug group, which is statistically significant $(P=0.005)$. This finding does not matched with Kingnorth et $\mathrm{al}^{(19)}$, and Horharin et $\mathrm{al}^{(18)}$, who reported no significant difference between both group in amount of analgesics used by the patients post operatively in spite of the lower amount of tablets used in mesh plug group rather than Lichtenstein group. There were no recurrence both for Lichtenstein and mesh with plug techniques. Bringman et $\mathrm{al}^{(21)}$ reported a late complication of recurrence with value of $1.9 \%$ for mesh plug and $0 \%$ for Lichtenstein. Frey et $\mathrm{al}^{(22)}$ reported a higher rate of recurrence in Lichtenstein group rather than mesh with plug group.

\section{Conclusion}

Compared to patients who received the Lichtenstein patch for inguinal hernia repair, patients who underwent the Mesh with Plug operation experienced more postoperative pain in the first 24 hours after the operation and consumed more postoperative analgesic medication. The rate of return to work and the hospital stay is similar in both groups. Despite that there is difference in post operative pain scoring, operative time and analgesic medication between both groups; there is no superiority between both groups in post operative complications, rehabilitation issues and recurrence rate.

\section{References}

1. Read RC. Herniology past, present and future. Hernia 2009; 13(6): 577-580.

2. Kingsnorth AN, leblanc KA. Management of abdominal hernias; http://www.hoepli.it/libro/management-ofabdominal-hernias/ 9781848828766 .html

3. Freeman ME, Smith SL. Inguinal hernia: open repair. In Hinder RA, Kelly KA, Sarr MG. (eds) Mayo Clinic Gastrointestinal surgery; 2004: 679-689.

4. Kux $M$, Fuchsjager $N$, Schemper $M$. Shouldice is superior to Bassini inguinal herniorrhaphy; Am. J. Surg; 1994: 168 (1):15-18.

5. Pation JF. A history of the treatment of hernia IN: Nyhus LM, Condon RE, eds. Hernia, $4^{\text {th }}$ ed. Philadelphia, Pennsylvania: JB Lippincott. 1995; 3-15.

6. Amid PK, shulman AG. Lichtenstein IL. Local anesthesia for anesthesia for inquinal hernia repair step-by-step procedure. Ann Surg. 1999; 220 (6): 735737.

7. Lichtenstein IL, Shulman AG, Amid PK: the tension -free repair of groin hernias, In: Nyhus LM, condon RE, (eds). Hernia ed. Philadelphia JE Lippincott. 2002: 237-247.

8. Rutkow IM, Robins AW. Tension free inguinal herniorrhaphy: a preliminary report on the "mesh-plug"technique. Surgery 1993; 114(1): 3-8.

9. Read RC, Yoder G. Recent trends in the management of inguinal herniation; Arch Surg; 1989; 124 (4):485-488.

10. Neumayer LA, Gawande AA, Wang J, et al. Proficiency of surgeon in inguinal hernia repair: Effect of experience and age: Ann Surg 2005; 242 (3):344-348.

11. Robbins AW, Rutkow IM, The mesh- 
plug hernioplasty. Surg Clin North Am; 1993:73 (3):501-512.

12. Amid PK, Shulman AG, Lichtenstein IL: selecting synthetic mesh for repair of groin hernia. Postgraduate general. Surgery; 2000; 4: 150.

13. Gilbert Al. An anatomic and functional Classification for the diagnosis and treatment of inguinal hernia. Am. J. surg. 1998; 157 (3): 331-333.

14. Rutkow IM, Robbins AW. Demographic, classificatory, and socioeconomic aspects of hernia repair in the United States. Surg Clin North Am; 1993: 73 (3): 413-426.

15. McCaffery M, Pasero CL. Pain: Clinical Manual, 2nd ed. St. Louis: Mosby; 1999:15-34.

16. Gilbert Al. The no-tension repair of groin hernias. In: Nyhus LM, condon RE (eds). Hernia, 4 th ed . Philadelphia, JB Lippincott. 2001: 248-9.

17. Saad A, Gaber M, Metawe A, Fathy M. Comparative Study between Lichtenstein Patch Hernioplasty Versus Tailored Plug and Patch Hernioplasty as A Treatment of Inguinal Hernia. JMRI, 2010; 31(1): 24-30.

18. Horharin P, Wilasrusmee C, Cherudchayaporn K, Pinyaku N, Phanpradi O, Phromsopha N. Comparative Study of Tailor-made Mesh Plug Herniorrhaphy versus Lichtenstein Herniorrhaphy versus Bassini Operation: A Prospective Clinical Trial. Asian J Surg. 2006; 29: 7478.

19. Kingsnorth AN, Christine S, Porter RN, et al. Lichtenstein patch or Perfix plugandpatch in inguinal hernia: A prospective double-blind randomized controlled trial of short-term outcome. Surgery. 2000; 127: 89-93.

20. Nienhuijs SW, van Oort I, Keemers-Gels ME, Strobbe LJ, Rosman C. Randomized clinical trial comparing the Prolene Hernia System, mesh plug repair and Lichtenstein method for open inguinal hernia repair. Br J Surg. 2005; 92 (1): 33-38.

21. Bringman $S$, Ramel $S$, Heikkinen $T$, Englund T, Westman B, Anderberg B.
Tension-Free Inguinal Hernia Repair: TEP Versus Mesh-Plug Versus Lichtenstein A Prospective Randomized Controlled Trial. Ann Surg. 2003. 237 (1): 142-147.

22. Frey DM, Wildisen A, Hamel CT, Zuber $M$, Oertli D, Metzger J. Randomized clinical trial of Lichtenstein's operation versus mesh plug for inguinal hernia repair. Br J Surg. 2007; 94(1):36-41. 